\title{
Impact of glucuronide interferences on therapeutic drug monitoring of posaconazole by tandem mass spectrometry
}

\author{
Ralf Krüger $^{1, *}$, Michael Vogeser ${ }^{2}$, \\ Stephan Burghardt ${ }^{3}$, Rita Vogelsberger ${ }^{1}$ and \\ Karl J. Lackner ${ }^{1}$ \\ ${ }^{1}$ Institute of Clinical Chemistry and Laboratory Medicine, \\ Medical Center of the Johannes Gutenberg University, \\ Mainz, Germany \\ ${ }^{2}$ Institute of Clinical Chemistry, Hospital of the University \\ of Munich, Munich, Germany \\ ${ }^{3}$ Chromsystems Instruments and Chemicals $\mathrm{GmbH}$, \\ Munich, Germany
}

\begin{abstract}
Background: Posaconazole is a novel antifungal drug for oral application intended especially for therapy of invasive mycoses. Due to variable gastrointestinal absorption, adverse side effects, and suspected drug-drug interactions, therapeutic drug monitoring (TDM) of posaconazole is recommended. Method: A fast ultra performance liquid chromatographytandem mass spectrometry (UPLC-MS/MS) method for quantification of posaconazole with a run-time $<3$ min was developed and compared to a LC-MS/MS method and HPLC method with fluorescence detection.

Results: During evaluation of UPLC-MS/MS, two earlier eluting peaks were observed in the MRM trace of posaconazole. This was only seen in patient samples, but not in spiked calibrator samples. Comparison with LC-MS/MS disclosed a significant bias with higher concentrations measured by LC-MS/MS, while UPLC-MS/MS showed excellent agreement with the commercially available HPLC method. In the LC-MS/MS procedure, comparably wide and left side shifted peaks were noticed. This could be ascribed to insource fragmentation of conjugate metabolites during electrospray ionisation. Precursor and product ion scans confirmed the assumption that the additional compounds are posaconazole glucuronides. Reducing the cone voltage led to disappearance of the glucuronide peaks. Slight modification of the LC-MS/MS method enabled separation of the main interference, leading to significantly reduced deviation.

Conclusions: These results highlight the necessity to reliably eliminate interference from labile drug metabolites for cor-

\footnotetext{
*Corresponding author: Dr. Ralf Krüger, Institute of Clinical Chemistry and Laboratory Medicine, Medical Center of the Johannes Gutenberg University, Langenbeckstr. 1, 55131 Mainz, Germany

E-mail: krueger@zentrallabor.klinik.uni-mainz.de Received April 15, 2010; accepted June 18, 2010; previously published online August 13, 2010
}

rect TDM results, either by sufficient separation or selective MS conditions. The presented UPLC-MS/MS method provides a reliable and fast assay for TDM of posaconazole. Clin Chem Lab Med 2010;48:1723-31.

Keywords: glucuronide; interference; liquid chromatography-tandem mass spectrometry (LC-MS/MS); metabolite; posaconazole; therapeutic drug monitoring; ultra performance liquid chromatography (UPLC).

\section{Introduction}

Triazole compounds are an established and effective class of antifungal drugs and are suited especially for therapy of systemic and superficial fungal infestations. Triazoles affect ergosterol biosynthesis of yeasts and fungi by specific inhibition of $14 \alpha$-demethylase (CYP51). Indications for therapeutic drug monitoring (TDM) comprise non-linear pharmacokinetics, high intra-individual metabolic variability, narrow therapeutic range, unexpected toxicity, and possible drug interactions (1-3). For triazole antifungals with an approval date before 2005, TDM methods have been developed for itraconazole and its main active metabolite, hydroxyitraconazole (4-7), as well as for voriconazole (8-11).

Posaconazole (Noxafil ${ }^{\circledR}$ ), a new antimycotic triazole drug for oral applications (Figure 1), was approved in 2005 in Europe and 2006 in the USA. Posaconazole is intended for therapy and prophylaxis of systemic and invasive mycoses, particularly in patients with impaired immune function due to immunosuppression, AIDS, or radiation therapy $[(12,13)$, and comment]. Posaconazole is suited especially for treatment of Candida and Aspergillus (14), but in contrast to itraconazole, it is also effective in cases with rare mycoses, such as fusarium (15) or zygomycetes (16). However, it has been reported that side effects are higher when compared to other triazoles $(17,18)$. TDM of posaconazole has been recommended when drug-drug interactions are suspected (2), and a clinical trial showed that higher posaconazole concentrations were associated with a higher number of responders to treatment of aspergillosis (14). Moreover, posaconazole metabolism shows pronounced individual variability of $38 \%-82 \%$ in cohorts of severely ill patients $(1,5,19)$. These variations have been ascribed primarily to differences in gastrointestinal drug absorption (20). Therefore, there is strong evidence that monitoring of plasma posaconazole concentrations is indicated, although further evaluation of the benefit of posaconazole TDM is certainly required. 
<smiles>[X]c1ccc([C@]2(Cn3cncn3)C[C@H](COc3ccc(N4CCN(c5ccc(-n6cnn([R])c6=O)cc5)CC4)cc3)CO2)c([X])c1</smiles><smiles></smiles>

Figure 1 Structure of the antimycotic drugs posaconazole and itraconazole, the active metabolite hydroxyitraconazole, and the internal standards ISTD1 (SCH 56984) and ISTD2 (R051012) that were used.

Currently, there is no consensus with respect to the therapeutic range of posaconazole. Moreover, standardization of assays for posaconazole quantification is another issue to be addressed. Various HPLC assays for quantification of posaconazole have been reported, applying either UV detection $(11,21,22)$, fluorescence detection (FLD) (23) or mass spectrometric detection (LC-MS) (24-29). However, inter-laboratory comparisons of different posaconazole assays have not been reported at present, and there is currently only limited availability of proficiency testing schemes.

An important issue affecting TDM methods are possible interferences, either from co-administered drugs or from metabolites of the parent drug. No evidence was found for formation of pharmacologically active metabolites of posaconazole (21). Posaconazole is metabolized essentially via glucuronidation by UDP-glucuronosyltransferase, resulting in mono- and di-glucuronides as the primary metabolites in plasma $(25,30)$. In-source fragmentation of labile glucuronides is known for other drugs, such as mycophenolic acid (31, 32), but no interfering metabolite peaks have been described so far in LC-MS assays for posaconazole TDM.

In this article, observations concerning metabolite interferences during TDM of posaconazole are reported. Interferences became apparent during development of a new, fast ultra performance liquid chromatography-tandem mass spectrometry (UPLC-MS/MS) method, and upon comparative analysis with a LC-MS/MS method under evaluation at another laboratory which showed significant quantitative differences. In contrast, no systematic deviations were found when compared with two independent HPLC methods using fluorescence detection (FLD). Identification of the interfering compounds by precursor and product scan experiments is described. Moreover, consequences for TDM of posaconazole are discussed, and precautionary measures for prevention of falsely increased results are suggested.

\section{Materials and methods}

\section{UPLC-MS/MS}

Chemicals and standards HPLC grade methanol (Merck, Darmstadt, Germany or Fisher Scientific, Schwerte, Germany) and HPLC grade water (Mallinckrodt-Baker, Deventer, Netherlands) were used as solvents. Formic acid and ammonium formate for mass spectrometry and $\mathrm{ZnCl}_{2}$ were obtained from Fluka/Sigma-Aldrich (Taufkirchen, Germany). Matrix-matched standards and controls from Chromsystems (Munich, Germany) were used for calibration and quality control. Two internal standards were used: ISTD1 was provided by Schering-Plough/Essex Pharma (Munich, Germany) and ISTD2 was obtained from Janssen Pharmaceuticals (Beerse, Belgium).

Sample preparation The protein precipitation solution, consisting of $40 \mathrm{~mL} \mathrm{MeOH}, 10 \mathrm{~mL} \mathrm{ZnCl} 2(0.2 \mathrm{M})$, and $350 \mu \mathrm{L}$ of the two internal standards $(100 \mathrm{mg} / \mathrm{L}$ in methanol each), was prepared fresh at least every 2 weeks. Three hundred microlitre of the methanolic precipitation solution was added to $100 \mu \mathrm{L}$ serum, vortexed intensively and centrifuged $10 \mathrm{~min}$ at $13,000 \mathrm{~g}$. Standards (three levels plus blank calibrator) and controls were treated identically. The supernatant was transferred to glass micro-vials and $10 \mu \mathrm{L}$ were injected for LC-MS analysis.

Instrumental LC-MS/MS analysis was performed using a Quattro Premier XE equipped with an Acquity UPLC system (Waters, Milford, MA, USA). A BEHC18 column $(50 \times 2.1 \mathrm{~mm})$ with $1.7 \mu \mathrm{m}$ particles was used in combination with a VanGuard precolumn with the same material (Waters, Milford, MA, USA). The flow rate was $0.6 \mathrm{~mL} / \mathrm{min}$ and the column temperature was $60^{\circ} \mathrm{C}$. Elution was achieved by linear gradient from $50 \%$ to $100 \%$ methanol in $0.8 \mathrm{~min}$. Hundred percentage methanol was held for another $0.8 \mathrm{~min}$, followed by equilibration with the starting conditions. Total run time was $2.6 \mathrm{~min}$. The divert valve was switched to waste until $0.8 \mathrm{~min}$. Both solvents contained $0.1 \%$ formic acid and $2 \mathrm{mM}$ ammonium formate. The latter is not necessary for the posaconazole assay, but identical solvents and columns were used also for analysis 
of immunosuppressants. No deleterious effects from the addition of ammonium salt were observed.

MS conditions were as follows: spray voltage $3.8 \mathrm{kV}$, desolvation gas $500 \mathrm{~L} / \mathrm{h}$, cone gas $50 \mathrm{~L} / \mathrm{h}$, desolvation temperature $350^{\circ} \mathrm{C}$, source temperature $100^{\circ} \mathrm{C}$, cone voltage $55 \mathrm{~V}$. The following transitions were monitored for MRM (dwell time was $30 \mathrm{~ms}$ ) in the UPLC-MS/MS method A: 687.3>600.3 (ISTD1); 701.3>614.3 (posaconazole); $\quad 705.3>392.3 \quad$ (itraconazole); $\quad 721.3>408.3$ (hydroxyitraconazole); 733.3 $>460.3$ (ISTD2). Alternative transitions were used in UPLC-MS/MS method B for ISTD1 $(687>669.3)$ and posaconazole $(701.3>683.3)$. Collision energy was $33 \mathrm{~V}$ for posaconazole and ISTD1, and $37 \mathrm{~V}$ for itraconazole, hydroxyitraconazole and ISTD2. Identical conditions were used for precursor and product scan experiments with a dwell time of $500 \mathrm{~ms}$.

\section{LC-MS/MS}

The method was performed essentially as described previously (28). It was comprised of a precipitation step, followed by online SPE and isocratic elution into the MS.

\section{HPLC-FLD}

A commercial kit from Chromsystems (Munich, Germany) was used for analysis of posaconazole, itraconazole and hydroxyitraconazole. Sample preparation was performed according to the kit instructions. Samples were analyzed in two different laboratories. In addition, an in-house method with similar parameters was used for comparison.

\section{Results}

\section{Evaluation of the UPLC-MS/MS method}

A fast UPLC-MS/MS method for routine TDM of posaconazole with a run-time below 3 min was established. The main benefits of UPLC are the enhanced duty-cycle in combination with excellent separation efficiency and peak shape. Validation confirmed good performance of the assay, covering the desired concentration range. Imprecision and recovery was determined using spiked controls. Total imprecision was determined to be $10.9 \%$ at $251 \mu \mathrm{g} / \mathrm{L}, 8.3 \%$ at 1015 $\mu \mathrm{g} / \mathrm{L}$ and $7.3 \%$ at $4487 \mu \mathrm{g} / \mathrm{L}$ (for inter-assay and intra-assay imprecision compare Table 1). The mean values of the three concentration levels correspond to recoveries of $100.3 \%$, $96.7 \%$ and $107.1 \%$. The limit of detection was $12 \mu \mathrm{g} / \mathrm{L}$ [defined by a signal-to-noise ( $\mathrm{S} / \mathrm{N})$ ratio of 3:1], and the limit of quantification was $37 \mu \mathrm{g} / \mathrm{L}$ (S/N 9:1). Linear response was obtained between $25 \mu \mathrm{g} / \mathrm{L}$ and $3634 \mu \mathrm{g} / \mathrm{L}$, with a regression equation of $\mathrm{y}=(1.026 \pm 0.015) \times-(4.914 \pm 6.299)$ and $\mathrm{R}^{2}=0.9948$. Upon infusion experiments, reproducible and gradient-dependant intensities were observed, with almost constant analyte intensities in the elution range of posaconazole, implicating minimized impact of ion suppression (compare Figure 2A).

\section{Identification of glucuronide metabolites}

Upon starting the UPLC-MS/MS method evaluation, two earlier eluting peaks were observed in most patient samples (compare Figure 3A), albeit with varying intensities. Addi-

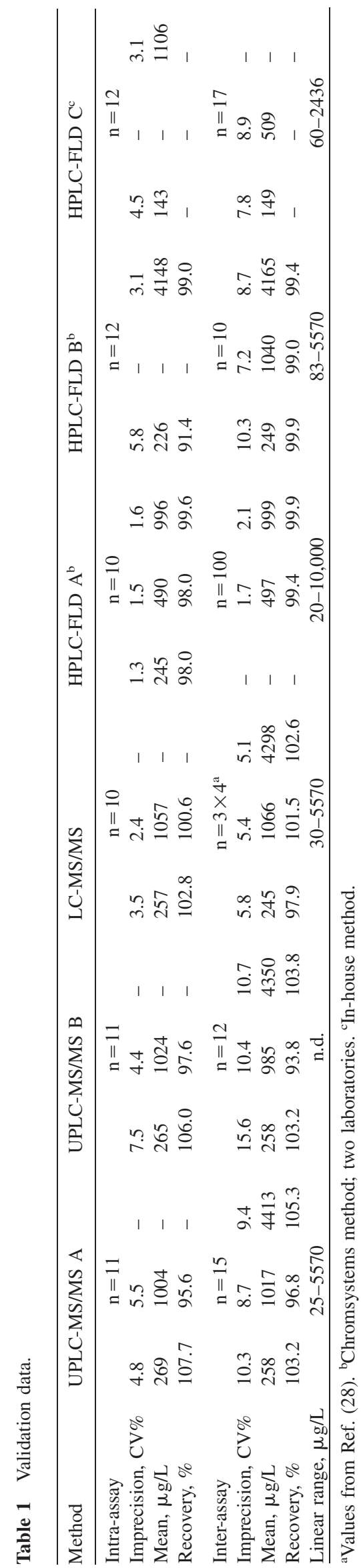



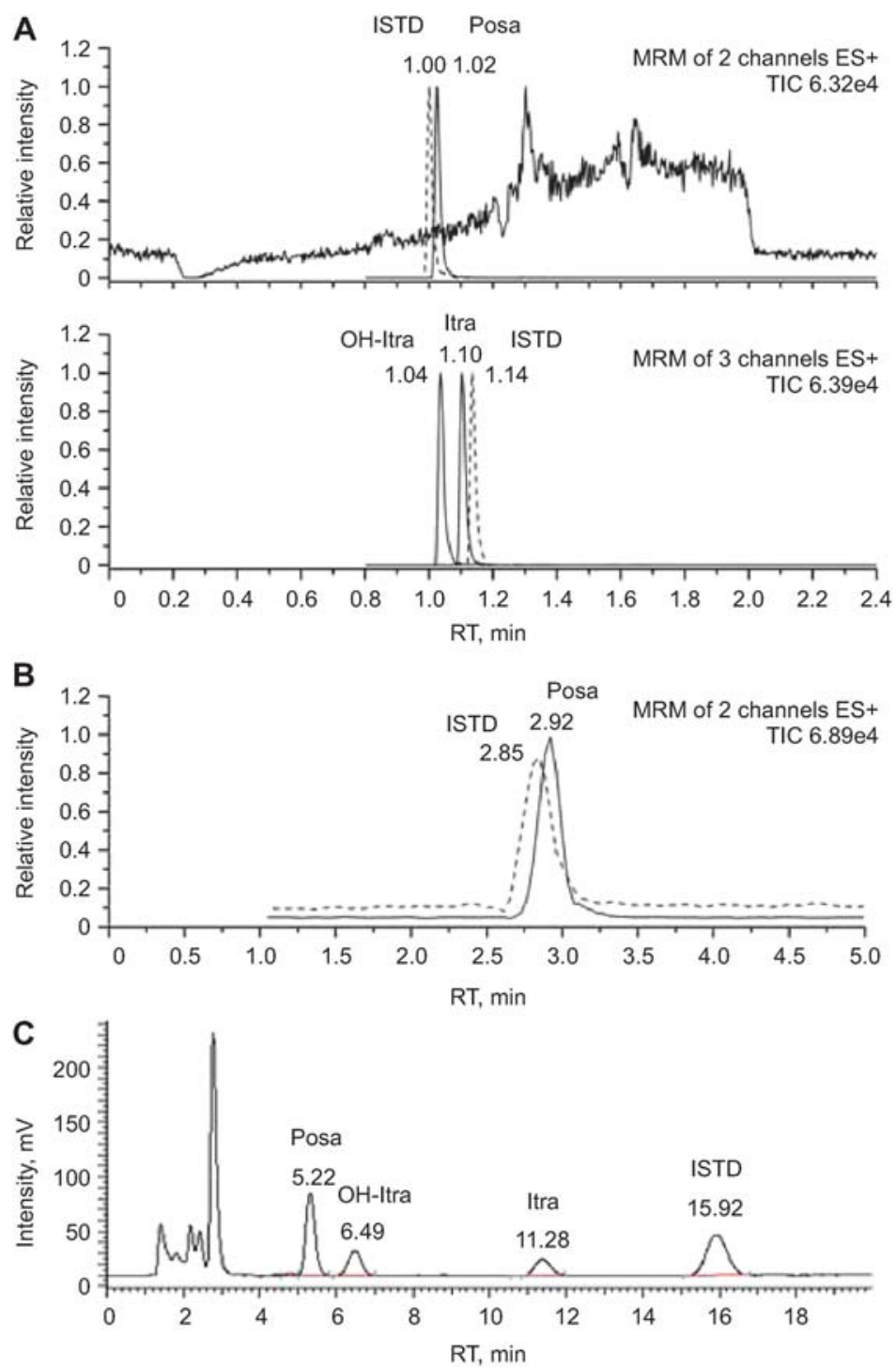

Figure 2 Example chromatograms (standards) of the new UPLC-MS/MS method and two of the assays used for comparative measurements. (A) UPLC-MS; upper panel: MRM traces posaconazole + ISTD1+infusion (ion suppression experiment); lower panel: MRM traces for itraconazole + hydroxyitraconazole + ISTD2; (B) LC-MS; MRM traces for posaconazole and ISTD1; (C) HPLC-FLD (Chromsystems).

tional, peaks were observed exclusively in post-dose patient samples, but not in spiked calibrators or controls. Moreover, a mean deviation of approximately $50 \%$ in patient samples was found following comparative measurements during evaluation of a LC-MS/MS method adapted from Ref. (28). Inspection of the chromatograms showed that peak width was wider and peak shape was left side shifted.

Our suspicion was that additional peaks and quantitative deviations could be caused by interference from conjugate metabolites, namely glucuronides (25, 30). Obviously, decomposition of metabolites and liberation of the parent drug in the ion source leads to the appearance of additional peaks in the MRM trace of posaconazole. In-source decomposition of labile glucuronides is well-known, as for example in the case of $\mathrm{MMF} /$ mycophenolate $(31,32)$. In order to confirm this assumption, parent scan and precursor scan experiments were performed on the UPLC-MS system. Traces
$A$ and B in Figure 3 show parent scans of the most intense fragment ions of posaconazole, m/z 614 and m/z 683. All three peaks were indeed detected in both traces. The respective mass spectra at the retention times (RT) $0.91 \mathrm{~min}$, $0.99 \mathrm{~min}$ and $1.03 \mathrm{~min}$ are shown in Figure 4A-C. The parent mass of posaconazole ( $\mathrm{m} / \mathrm{z} 701)$ appears in all spectra, but it is not the highest mass in the spectra of the two earlier eluting peaks at RT $0.91 \mathrm{~min}$ and $0.99 \mathrm{~min}$. Instead, another precursor ion at $\mathrm{m} / \mathrm{z} 877$ is clearly visible, in accordance with the expected mass of a glucuronide metabolite (mass difference of 176). This peak is absent in the spectrum obtained at RT $1.03 \mathrm{~min}$, the expected elution time of posaconazole.

A cross check was performed by product scan experiments. Figure 3D shows the product scan of $\mathrm{m} / \mathrm{z} 877$, the mass of the intact protonated glucuronide. The first two peaks appear in this experiment exclusively, in accordance with the assumption that only these are caused by glucuro- 

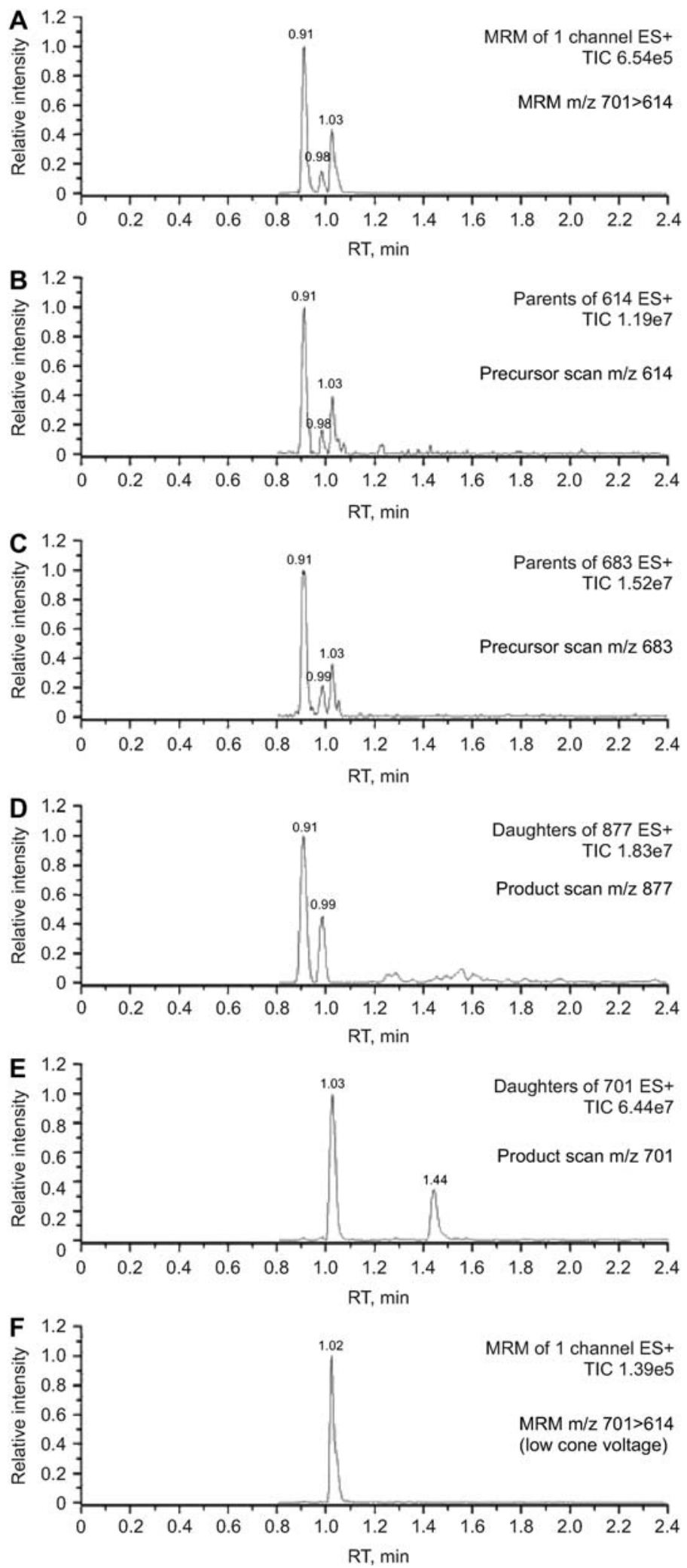

Figure 3 UPLC-MS chromatograms for a patient sample showing the detection of glucuronide metabolites. The peaks at RT 0.91 min and RT 0.99 min correspond to posaconazole metabolites, whereas the peak at RT 1.03 min corresponds to the free drug. The peak at RT $1.44 \mathrm{~min}$ in trace $\mathrm{E}$ is not a posaconazole derivative, since the corresponding mass spectrum is completely different.

(A) MRM trace 701 >614; (B) precursor scan of $\mathrm{m} / \mathrm{z} 614$; (C) precursor scan of $\mathrm{m} / \mathrm{z} 683$; (D) product scan of $\mathrm{m} / \mathrm{z} 877$ (glucuronide);

(E) product scan of m/z 701 (free drug); (F) MRM trace 701 $>614$ at lower cone voltage (20 V instead of $55 \mathrm{~V}$ ). 

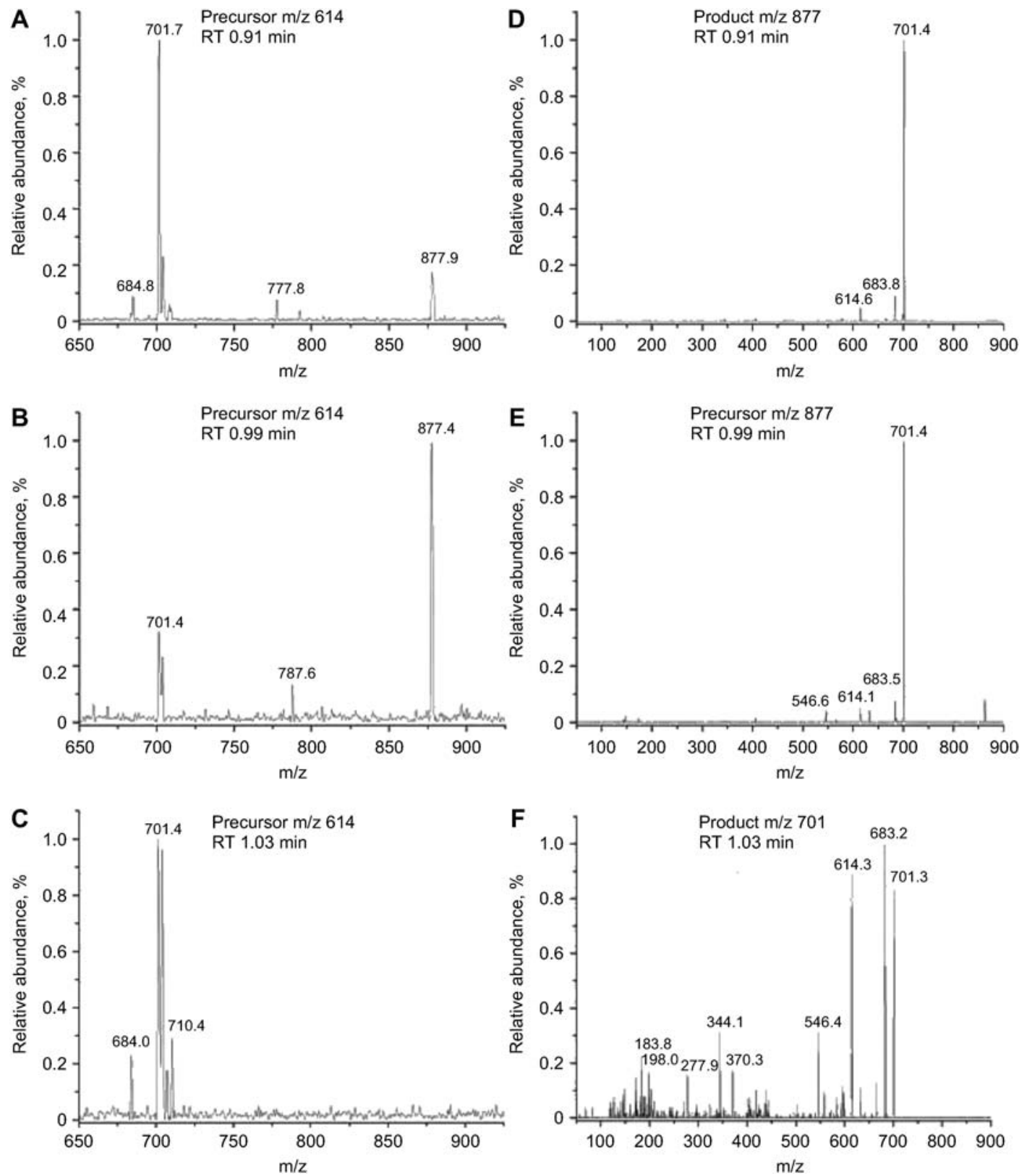

Figure 4 MS/MS spectra from peaks in the chromatograms of Figure 3 which confirm that the additional peaks in the MRM trace are caused by glucuronide metabolites.

(A) Mass spectrum at RT $0.91 \mathrm{~min}$ in the precursor scan of $\mathrm{m} / \mathrm{z} 614$ (trace B in Figure 3); (B) mass spectrum at RT $0.99 \mathrm{~min}$ in the precursor scan of m/z 614 (trace B in Figure 3); (C) mass spectrum at RT 1.03 min in the precursor scan of m/z 614 (trace B in Figure 3); (D) mass spectrum at RT $0.91 \mathrm{~min}$ in the product scan of $\mathrm{m} / \mathrm{z} 877$ (trace D in Figure 3); (E) mass spectrum at RT $0.99 \mathrm{~min}$ in the product scan of m/z 877 (trace D in Figure 3); (F) mass spectrum at RT $1.03 \mathrm{~min}$ in the product scan of m/z 701 (trace E in Figure 3).

nide metabolites. The corresponding mass spectra are depicted in Figure 4D and E. In both cases, the main fragment is the ion with $\mathrm{m} / \mathrm{z} 701$, which is the mass of the protonated parent drug posaconazole. In addition, to the main peak, fragment ions at m/z 614 and m/z 683 are also detected with low intensity. These correspond to the most abundant fragments of posaconazole, which have been selected for the MRM experiments (compare spectrum in Figure 4F). These observations give clear proof that the two earlier eluting compounds are indeed posaconazole glucuronides. In con- trast, the product ion scan of $\mathrm{m} / \mathrm{z} 701$ (Figure 3E) shows nearly exclusively the posaconazole peak at RT 1.03 , which can be identified by its product mass spectrum depicted in Figure 4F. However, small peaks are also visible at RT $0.91 \mathrm{~min}$ and $0.99 \mathrm{~min}$, probably due to release of parent drug from the respective glucuronides in the ion source, which explains the additional peaks in the posaconazole MRM trace. It should be noted that the peak at RT 1.44 is not a posaconazole derivative, since the corresponding product mass spectrum is completely different. The identification 
of glucuronides was supported also by chemical hydrolysis experiments using $\mathrm{NaOH}$ which resulted in a significant decrease in the metabolite peaks (factor 5-8 after $1.5 \mathrm{~h}$ incubation at $37^{\circ} \mathrm{C}$ ).

\section{Elimination of glucuronide interferences}

Eliminating glucuronide interference is mandatory for the correct determination of drug plasma concentrations. Interfering peaks were clearly separated with UPLC-MS/MS, preventing falsely increased results.

Alternatively, the influence of the metabolites can be reduced significantly by choosing softer conditions in the ion source. The main effect was observed by reducing the cone voltage. This value is not only relevant for ion extraction, but also influences the extent of ion desolvation and fragmentation in the ion source. Reduction of the cone voltage from $55 \mathrm{~V}$ to $20 \mathrm{~V}$ led to almost complete disappearance of the metabolite peaks (see Figure 3F). However, there was decreased intensity of the posaconazole peak by a factor of approximately 2-3 (compare Figure 3A). Since the noise level was reduced by a similar factor, the signal-to-noise ratio was not compromised. Thus, even with insufficient separation, interferences can be minimized and correct quantification is possible, provided soft conditions in the ion source are chosen.

Baseline separation of posaconazole from the main metabolite was also achieved using the LC-MS method by slight modification of chromatographic parameters, including smoothing and integration settings. This provided proof that the glucuronide interference caused the asymmetrical peak shape when applying the initial settings.

\section{Assay comparison}

After method adjustments, the correlation between the MSbased methods $(\mathrm{n}=48)$ appeared to be significantly improved with $\mathrm{R}^{2}=0.9768$ and linear regression of $y=(1.0726 \pm 0.0645) \times+(1.8610 \pm 10.2335)$. However, the second interference could not be separated without major method changes, indicated by the residual mean deviation of $10.3 \%$ as assessed using the Bland-Altmann plot. Assay modifications aiming to eliminate this remaining interference were initiated.

It should be mentioned that choice of the internal standard and MRM transition was essential for application of UPLCMS. Two different internal standards were tested, and the itraconazole analogue (ISTD2) gave systematically higher values (mean deviation 17\%) compared to the posaconazole analogue (ISTD1). The difference was even more pronounced (mean deviation 30\%) when compared to the HPLC-FLD methods which use the identical ISTD2 (correlations are shown in Supplementary Figure 1). Moreover, higher imprecision was observed when using the transition $701>683$ (loss of water, UPLC method B) instead of the transition $701>614$ (UPLC method A). This effect was not observed when using online-SPE (method 2), which generally showed a better CV.
Six samples gave no detectable signal with HPLC-FLD. Thus, 42 samples in the concentration range $31-1437 \mu \mathrm{g} / \mathrm{L}$ were available for general method comparison between HPLC and UPLC-MS (see Figure 5). The correlation was excellent $\left(\mathrm{R}^{2}=0.994\right)$ with linear regression of $\mathrm{y}=(1.046 \pm 0.039) \times+(2.756 \pm 7.446)$ and a mean deviation of $4 \%$ (Bland-Altmann). Good correlation $\left(\mathrm{R}^{2}>0.99\right)$ was also obtained between two HPLC methods, even though the in-house method utilizes aqueous instead of matrix-matched standards. Basic validation data for all methods are shown in Table 1, and detailed correlation data can be found in Supplementary Table 1.

\section{Discussion}

The results of the present investigation substantiate the importance of standardization of TDM assays. Appropriate correlation was demonstrated after matrix-matched standards become available. Moreover, the present example underlines that it is necessary to be aware of pitfalls which may be related to standards, internal standards or isobaric interfer-
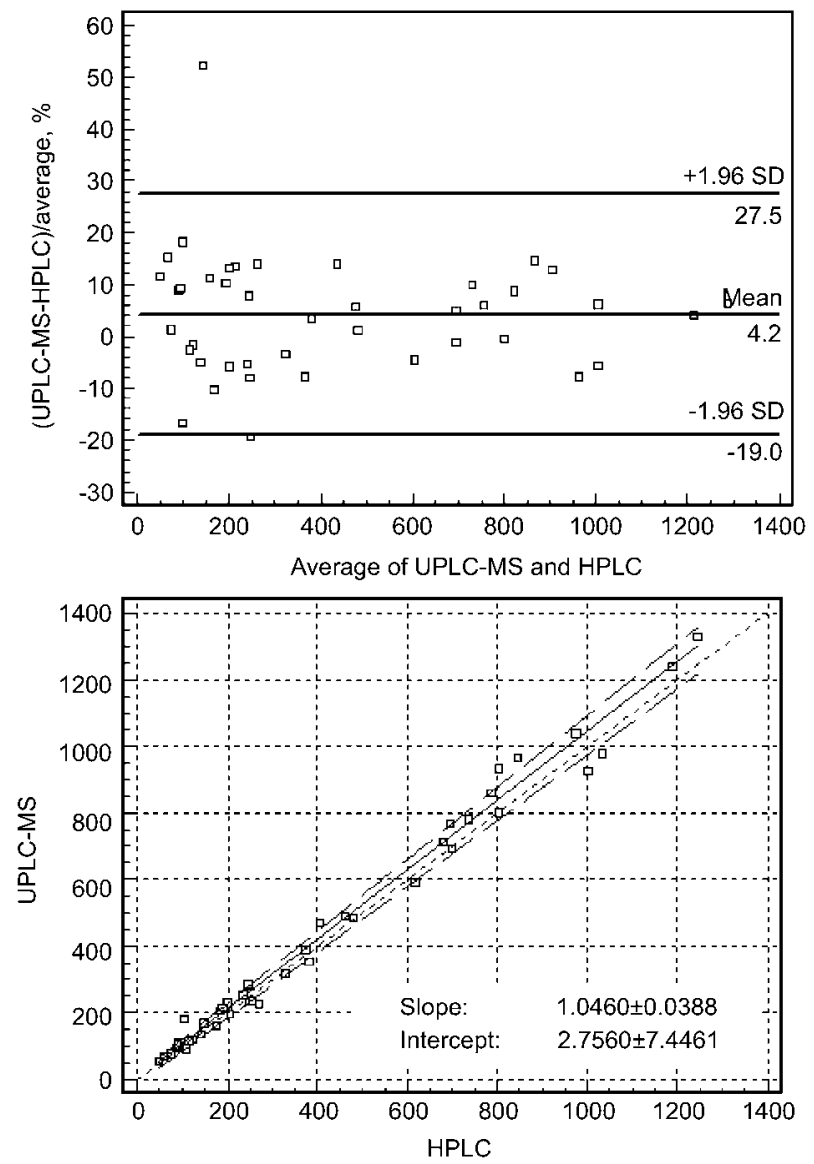

Figure 5 Correlation of UPLC-MS with HPLC assays (mean, $n=2$, two laboratories) for determination of serum posaconazole concentrations.

ISTD1 was used in case of UPLC-MS, whereas ISTD2 was used in case of HPLC. 
ences, such as co-administered drugs and metabolites of the target molecule. In particular, it is critical that a sufficient number of real-life patient samples be check for the occurrence of interference at an early stage of method evaluation. Upon development of the posaconazole TDM methods, we observed adverse effects due to (i) the choice of the internal standard (ii) the MRM transition in UPLC-MS/MS and (iii) in-source decay of analyte metabolites, namely glucuronides.

The itraconazole analogue (ISTD2) can be successfully applied to HPLC analysis, but it is not suited to LC-MS. These systematic deviations underline the need for almost identical structures of analyte and internal standard to compensate ionization effects. Unfortunately, the posaconazole analogue (ISTD1) is currently no longer available. Hopefully, the growing demand for reliable LC-MS assays will eventually help solve this problem. Moreover, the choice of MRM transition had a clear influence on method performance and imprecision if no online-SPE was applied (see Table 1). Apparently, the MRM transition 701>683 (loss of water) is less specific compared to $701>614$, as may be expected.

However, the most relevant observation concerns the influence of metabolite interferences. The results are a striking example that conjugate metabolites, such as glucuronides can appear in the MRM trace of the analyte due to in-source decay and may thus impair TDM results. Usually, those interferences can be safely excluded by careful adjustment of instrument conditions, such as chromatography, tuning parameters or peak processing settings.

In principal, the described interferences might even have been predicted because the MS/MS spectra of the glucuronides obtained during metabolism studies showed the mass of the parent drug in the MS/MS spectra with high intensity. However, none of the developed posaconazole TDM assays published to date account for this effect. Short chromatography is preferred in TDM assays in order to enhance turn around time, which leads to a higher risk of insufficient separation of metabolites. In contrast, extended chromatographic separations are usually employed during drug discovery and pharmacokinetic characterisation of drug candidates, which minimizes the risk that metabolites are overlooked.

Online-SPE is another item which needs to be considered. Online-SPE improves signal stability by reducing ion suppression effects, and allows injection of larger sample volumes and analyte enrichment. However, elution from the SPE cartridge and subsequent chromatographic separation and elution from the analytical column is often achieved using isocratic conditions, since only one gradient pump is necessary for this configuration. As a consequence, chromatographic resolution is inherently lower compared to methods employing gradient separations, irrespective of whether HPLC or UPLC are applied. Therefore, the risk of overlooking metabolite interferences is greater with onlineSPE and isocratic elution.

Our observation underscores that in-source transformation is an important potential pitfall in short LC-MS/MS methods applied to TDM. Reliable elimination of interferences depends to a great degree on individual instrument configuration and settings, such as MS tuning or chromatographic parameters. Moreover, careful evaluation of the peak shape of patient samples is mandatory and may help detect unidentified interferences or other adverse effects. Also, if short TDM methods are desired, it seems inevitable that samples be thoroughly screened for the occurrence of metabolites during early method evaluation, for example by analyzing a sufficiently high number of patient samples with a longer LC gradient than usually applied for TDM.

In summary, the presented fast UPLC-MS/MS method allows for reliable TDM of posaconazole without interference from co-eluting glucuronides. In addition, the results are consistent with those obtained by independent methods.

\section{Conflict of interest statement}

Authors' conflict of interest disclosure: The authors stated that there are no conflicts of interest regarding the publication of this article.

Research funding: None declared.

Employment or leadership: None declared.

Honorarium: None declared.

\section{References}

1. Smith J, Andes D. Therapeutic drug monitoring of antifungals: pharmacokinetic and pharmacodynamic considerations. Ther Drug Monit 2008;30:167-72.

2. Worth LJ, Blyth CC, Booth DL, Kong DC, Marriott D, Cassumbhoy $\mathrm{M}$, et al. Optimizing antifungal drug dosing and monitoring to avoid toxicity and improve outcomes in patients with haematological disorders. Int Med J 2008;38:521-37.

3. Goodwin ML, Drew RH. Antifungal serum concentration monitoring: an update. J Antimicrob Chemother 2008;61:17-25.

4. Cox SK, Orosz S, Burnette J, Frazier D. Microassay for determination of itraconazole and hydroxyitraconazole in plasma and tissue biopsies. J Chromatogr B 1997;702:175-80.

5. Gubbins PA, Gurley BJ, Bowman J. Rapid and sensitive high performance liquid chromatographic method for the determination of itraconazole and its hydroxy-metabolite in human serum. J Pharm Biomed Anal 1998;16:1005-12.

6. Koks CH, Sparidans RW, Lucassen G, Crommentuyn KM, Beijnen JH. Selective high-performance liquid chromatographic assay for itraconazole and hydroxyitraconazole in plasma from human immunodeficiency virus-infected patients. J Chromatogr B 2002;767:103-10.

7. Redmann S, Charles BG. A rapid HPLC method with fluorometric detection for determination of plasma itraconazole and hydroxyitraconazole concentrations in cystic fibrosis children with allergic bronchopulmonary aspergillosis. Biomed Chromatogr 2006;20:343-8.

8. Keevil BG, Newman S, Lockhart S, Howard SJ, Moore CB, Denning DW. Validation of an assay for voriconazole in serum samples using liquid chromatography-tandem mass spectrometry. Ther Drug Monit 2004;26:650-7.

9. Egle H, Trittler R, König A, Kümmerer A. Fast, fully automated analysis of voriconazole from serum by LC-LC-ESI-MS-MS with parallel column-switching technique. J Chromatogr B 2005; 814:361-7. 
10. Vogeser M, Schiel X, Spöhrer U. Quantification of voriconazole in plasma by liquid chromatography-tandem mass spectrometry. Clin Chem Lab Med 2005;43:730-4.

11. Chhun S, Rey E, Tran A, Lortholary O, Pons G, Jullien V. Simultaneous quantification of voriconazole and posaconazole in human plasma by high-performance liquid chromatography with ultra-violet detection. J Chromatogr B 2007;852:223-8.

12. Torres HA, Hachem RY, Chemaly RF, Kontoyiannis DP, Raad II. Posaconazole: a broad-spectrum triazole antifungal. Lancet Infect Dis 2005;5:775-85.

13. Nagappan V, Deresinski S. Posaconazole: a broad-spectrum triazole antifungal agent. Clin Infect Dis 2007;45:1610-7.

14. Walsh TJ, Raad I, Patterson TF, Chandrasekar P, Donowitz GR, Graybill R, et al. Treatment of invasive aspergillosis with posaconazole in patients who are refractory to or intolerant of conventional therapy: an externally controlled trial. Clin Infect Dis 2007;44:2-12.

15. Raad II, Hachem RY, Herbrecht R, Graybill JR, Hare R, Corcoran $\mathrm{G}$, et al. Posaconazole as salvage treatment for invasive fusariosis in patients with underlying hematologic malignancy and other conditions. Clin Infect Dis 2006;42:1398-403.

16. Diekema DJ, Messer SA, Hollis RJ, Jones RN, Pfaller MA. Activities of caspofungin, itraconazole, posaconazole, ravuconazole, voriconazole, and amphotericin B against 448 recent clinical isolates of filamentous fungi. J Clin Microbiol 2003;41: 3623-6.

17. Cornely OA, Maertens J, Winston DJ, Perfect J, Ullmann AJ, Walsh TJ, et al. Posaconazole vs. fluconazole or itraconazole prophylaxis in patients with neutropenia. N Engl J Med 2007; 356:348-59.

18. Ullmann AJ, Lipton JH, Vesole DH, Chandrasekar P, Langston A, Tarantolo SR, et al. Posaconazole or fluconazole for prophylaxis in severe graft-versus-host disease. N Engl J Med 2007;356:335-47.

19. Ullmann AJ, Cornely OA, Burchardt A, Hachem R, Kontoyiannis DP, Töpelt P, et al. Pharmacokinetics, safety, and efficacy of posaconazole in patients with persistent febrile neutropenia or refractory invasive fungal infection. Antimicrob Agents Chemother 2006;50:658-66.

20. Courtney R, Wexler D, Radwanski E, Lim J, Laughlin M. Effect of food on the relative bioavailability of two oral formulations of posaconazole in healthy adults. Br J Clin Pharmacol 2003;57:218-22.

21. Kim H, Kumari P, Laughlin M, Hilbert MJ, Indelicato SR, Lim $\mathrm{J}$, et al. Use of high-performance liquid chromatographic and microbiological analyses for evaluating the presence or absence of active metabolites of the antifungal posaconazole in human plasma. J Chromatogr A 2003;987:243-8.

22. Störzinger D, Swoboda S, Lichtenstern C, Müller C, Weigand MA, Hoppe-Tichy T. Development and validation of a highperformance liquid chromatography assay for posaconazole utilizing solid-phase extraction. Clin Chem Lab Med 2008; 46:1747-51.

23. Neubauer W, König A, Bolek R, Trittler R, Engelhardt M, Jung $\mathrm{M}$, et al. Determination of the antifungal agent posaconazole in human serum by HPLC with parallel column-switching technique. J Chromatogr B 2009;877:2493-8.

24. Feng W, Liu H, Chen G, Malchow R, Bennett F, Lin E, et al. Structural characterization of the oxidative degradation products of an antifungal agent SCH 56592 by LC-NMR and LC-MS. J Pharm Biomed Anal 2001;25:545-57.

25. Krieter P, Flannery B, Musick T, Gohdes M. Disposition of posaconazole following single-dose oral administration in healthy subjects. Antimicrob Agents Chemother 2004;48:3543-51.

26. Shen JX, Krishna G, Hayes RN. A sensitive liquid chromatography and mass spectrometry method for the determination of posaconazole in human plasma. J Pharm Biomed Anal 2007;43:228-36.

27. Shen JX, Tama CI, Hayes RN. Evaluation of automated micro solid phase extraction tips ( $\mu$-SPE) for the validation of a LC-MS/MS bioanalytical method. J Chromatogr B 2006; 843:275-82.

28. Vogeser M, Rieger C, Ostermann H, Spöhrer U. A routine method for the quantification of the novel antimycotic drug posaconazole in plasma using liquid chromatography-tandem mass spectrometry. Clin Chem Lab Med 2009;47:579-84.

29. Cunliffe JF, Noren CF, Hayes RN, Clement RP, Shen JX. A high-throughput LC-MS/MS method for the quantitation of posaconazole in human plasma: implementing fused core silica liquid chromatography. J Pharm Biomed Anal 2009;50:4652.

30. Ghosal A, Hapangama N, Yuan Y, Achanfuo-Yeboah J, Iannucci R, Chowdhury S, et al. Identification of human UDP-glucuronosyltransferase enzyme(s) responsible for the glucuronidation of posaconazole (Noxafil). Drug Metab Dispos 2004;32: 267-71.

31. Vogeser M, Zachoval R, Spöhrer U, Jacob K. Potential lack of specificity using electrospray tandem-mass spectrometry for the analysis of mycophenolic acid in serum. Ther Drug Monit 2001;23:722-4.

32. Kuhn J, Prante C, Kleesiek K, Götting C. Measurement of mycophenolic acid and its glucuronide using a novel rapid liquid chromatography-electrospray ionization tandem mass spectrometry assay. Clin Biochem 2009;4283-90. 